Pacific Journal of Mathematics

A FORM OF THE MOMENT PROBLEM FOR LIE GROUPS 


\title{
A FORM OF THE MOMENT PROBLEM FOR LIE GROUPS
}

\author{
Reese T. Prosser
}

\begin{abstract}
A form of the Hamburger moment problem for the real line is generalized and solved for an arbitrary Lie group. The solution relates the unitary representations of the Lie group to certain symmetric representations of the associated universal enveloping algebra.
\end{abstract}

The celebrated Hamburger moment problem for the real line asks: Which linear functionals on the algebra of polynomials can be realized by integrating these polynomials against a fixed positive measure ? Hamburger's classic solution shows that a linear functional has this property if and only if it assigns positive values to positive polynomials $[6,10]$.

By taking Fourier transforms, we may rephrase the problem as follows: Which linear functionals on the algebra of distributions supported at the origin can be realized by evaluating these distributions at a fixed function of positive type? The solution shows that a linear functional has this property if and only if it assigns positive values to distributions of positive type.

In this latter form we may extend the problem to an arbitrary Lie group, and search for a solution in the same form. Here we confirm that this extended problem does indeed admit such a solution (Theorem 3). We then reformulate the result in terms of the space of unitary representations of the group to give a less satisfactory extension of the problem in its original form (Theorem 4). In the process we find that we can characterize the symmetric representations of the associated Lie algebra which can be "lifted" to unitary representations of the group (Theorem 5). As in the classical case [1], the heart of the matter lies in showing that positive linear functionals on the moment algebra are necessarily continuous in an appropriate sense (Corollary 2).

The algebra of distributions of compact support. In order to formulate the problem on a given Lie group, we shall need an algebraic structure containing both the Lie group and its Lie algebra. For this purpose we choose the algebra of all distributions with compact support defined on the group. The properties of this algebra which we shall use are all obtained by direct extensions of arguments developed by Laurent Schwartz in his investigations of distribution defined on $R^{n}[9$, also $2,3,4]$ and are only summarized here. 
Let $G$ be a Lie group and $g$ its Lie algebra. Denote by $E^{(k)}$ the space of all $k$-times continuously-differentiable complex-valued functions defined on $G$, topologized by uniform convergence of all derivatives on all compacta; and by $E$ the projective limit of all infinitely differentiable functions, with the projective limit topology. Denote by $E^{(k) \prime}$ the dual space of all complex-valued distributions of order of most $k$ with compact support on $G$, topologized with the strong dual topology; and by $E^{\prime}$ the inductive limit of all distributions with compact support on $G$, topologized with the dual inductive limit topology. Then $E$ and $E^{\prime}$ are both Montel spaces, and each is the strong dual of the other. In particular, every linear functional on $E^{\prime}$ which is bounded on the bounded subsets of $E^{\prime}$ is necessarily continuous on $E^{\prime}$ and so must lie in $E$. The bounded subsets of $E^{\prime}$ are relatively compact; moreover, every bounded subset of $E^{\prime}$ is contained and bounded in $E^{(k) \prime}$ for some $k$ [cf. 11, Theorem 34.4].

Denote further by $E_{K}$, where $K$ is a compact subset of $G$, the quotient space of $E$ module the subspace of functions vanishing, together with all of their derivatives, on $K$; and by $E_{K}^{\prime}$ the dual subspace of $E^{\prime}$ consisting of distributions whose supports be in $K$. Then as $K$ runs through the family of all compact subsets of $G$, directed by inclusion, $E$ is obtained as the strict projective limit, and $E^{\prime}$ as the strict inductive limit, of $E_{K}$ and $E_{K}^{\prime}$, respectively. In particular, it follows that every bounded set in $E^{\prime}$ is contained in $K_{K}^{\prime}$ for some $K$ [cf. 11, Theorem 34.4].

The space $E^{\prime}$ admits an associative multiplication, defined by convolution over $G$ and denoted here simply by juxtaposition, such that the mapping $\{s, t\} \rightarrow s t$ is continuous jointly in $s$ and $t$. Under the multiplication, $E^{\prime}$ becomes a Montel algebra with unit $e$ (the point measure at the origin of $G$ ) which contains:

(a) an isomorphic image of the Lie group $G$ as the group of point measures under convolution,

(b) an isomorphic image of the Lie algebra $g$ as the algebra of tangent vectors at the origin of $G$ under the Lie product.

Moreover, both of these isomorphisms are topological. We denote by $A$ the associative subalgebra of $E^{\prime}$ generated algebraically by $G$, and by $U$ the associative subalgebra generated algebraically by $g$ and $e$. Then $A$ may be identified with the algebraic group algebra of $G$ and $U$ with the universal enveloping algebra of g. $A$ is dense in $E^{(0)}$, and hence in $E^{\prime}$, while $U$ coincides with $E_{\{e\}}$ '

In terms of the multiplication in $E^{\prime}$ we can describe the distributions of order $k$ as $k$-fold derivatives of distributions of order 0 , or Radon measures, on $G$. More precisely for every bounded set $B$ in $E^{(k) \prime}$, and hence in $E^{\prime}$, there is a bounded set $C$ in $E^{(0) \prime}$ and a finite 
set $D$ (consisting in fact of monomials in the generators of $\mathfrak{g}$ ) in $U^{(k)}=U \cap E^{(k) \prime}$, respectively, such that every $t \in B$ can be expressed in the form $t=\sum d_{j} m_{i}$ with $m_{i} \in C$ and $d_{i} \in D$ [11, Theorem 34.5]. Thus $E^{(k) \prime}$ is spanned by $U^{(k)} E^{(0) \prime}$.

Order properties. The space $E$ admits an involution ${ }^{*}: \varphi \rightarrow \varphi^{*}$ defined by the formula $\varphi^{*}(g)=\overline{\phi\left(g^{-1}\right)}, g \in G$. This involution is conjugate-linear and bicontinuous in the topology of $E$. We denote by $H$ the set of symmetric functions $\varphi$, for which $\varphi^{*}=\varphi$, and by $P$ the set of functions $\varphi$ of positive type, for which $\sum_{1 j} \lambda_{i} \bar{\lambda}_{j} \varphi\left(g_{i} g_{i}^{-1}\right) \geqq 0$, $\lambda_{i} \in C, g_{i} \in G$. Then $H$ is a closed real-linear subspace of $E$, and $P$ is a closed convex cone in $H$. Moreover, we know that $P \cap(-P)=$ $\{0\}$ (since if $\varphi \in P$ then $|\varphi(g)| \leqq \varphi(e), g \in G$ ) and that $P+(-P)$ is dense in $H$ [cf. 7, p. 142].

The space $E$ also admits an involution *: $t \rightarrow t^{*}$, induced by the involution in $E$ according to the formula $t^{*}(\varphi)=\overline{t\left(\varphi^{*}\right)}$. This involution is a conjugate-linear anti-automorphism, bicontinuous in the topology of $E^{\prime}$. Under this involution, $E^{\prime}$ becomes a Montel *-algebra which contains both $A$ and $U$ as *-subalgebras.

We denote by $H^{\prime}$ the set of symmetric elements $t \in E^{\prime}$ for which $t^{*}=t$, and by $P^{\prime}$ the set of elements $t$ of positive type, for which $t(\varphi) \geqq 0$ for all $\varphi \in P$. All elements of the form $t t^{*}$ lie in $P^{\prime}$ and convex combinations of such elements are dense in $P^{\prime}$. Every element $t$ of $P^{\prime}$ has the form $t=t_{r}+i t_{i}$, where $t_{r}=\frac{1}{2}\left(t+t^{*}\right)$ and $t_{i}=\frac{1}{2} i$ $\left(t-t^{*}\right)$ lie in $H^{\prime}$, and every element $t$ of $H^{\prime}$ has the form $t=t_{p}-t_{n}$, where $t_{p}=\frac{1}{4}(t+e)^{2}$ and $t_{n}=\frac{1}{4}(t-e)^{2}$ lie in $P^{\prime}$. Hence, $P^{\prime}+\left(-P^{\prime}\right)=$ $H^{\prime}$, and $H^{\prime}+i H^{\prime}=E$. Since $P+(-P)$ is dense in $H$, it follows that $P^{\prime} \cap\left(-P^{\prime}\right)=\{0\}$. Finally, since multiplication is jointly continuous in $E^{\prime}$, it follows that $P^{\prime}$ is invariant under all mappings of the form $t \rightarrow s t s^{*}$, where $s \in E^{\prime}$.

The cones $P$ and $P^{\prime}$ are dual cones, in the sense that $\varphi \in P$ if and only if $t(\varphi) \geqq 0$ for all $t \in P^{\prime}$, and $t \in P^{\prime}$ if and only if $t(\varphi) \geqq 0$ for all $\varphi \in P$. In terms of these cones we can define dual partial orderings in $H$ and $H^{\prime}$ by putting $\theta \leqq \varphi$ if and only if $\varphi-\theta \in P$, and $s \leqq t$ if and only if $t-s \in P^{\prime}$. Then these orderings are compatible with the algebra and topology of $H$ and $H^{\prime}$ and exhibit all the usual ordering properties [9]. In particular, if $s, t \in E^{\prime}$, then $(s+t)(s+t)^{*} \leqq(s+t)(s+t)^{*}+(s-t)(s-t)^{*}=2\left(s s^{*}+t t^{*}\right) . \quad$ Вy iterating this argument, we find that if $t_{1}, \cdots, t_{n} \in E^{\prime}$, then $\left(\Sigma t_{i}\right)$ $\left(\Sigma t_{i}\right)^{*} \leqq 2^{n}\left(\Sigma t_{i} t_{i}^{*}\right)$.

In terms of the ordering in $H$, we can now identify the bounded sets of $E^{\prime}$ as follows:

LEMma 1. A subset $B$ of $E^{\prime}$ is bounded if and only if there exist 
(a) a compact subset $K$ of $G$, and (b), a single element $d$ in $U \cap P^{\prime}$, such that $B \subset E_{K}{ }^{\prime}$, and for all $t \in B$ we have

$$
\begin{aligned}
& -d \leqq t_{r} \leqq+d \\
& -d \leqq t_{i} \leqq+d
\end{aligned}
$$

Proof. Suppose first that $B$ is bounded. Then we already know that $B \subset E_{K}^{\prime} \cap E^{(k) \prime}$ for some compact subset $K$ of $G$ and integer $k$. Now for any $t \in E^{\prime}$ we have $2 t_{r}=t+t^{*} \leqq t t^{*}+e$, and similarly for $-2 t_{r}$ and $\pm 2 t_{i}$. Hence it suffices to show that if $t \in B$, then there exists a single element $d \in U$ such that $t t^{*} \leqq d$.

Suppose first that $B \subset E^{(0) '}$. Then every $m \in B$ is a finite measure supported in $K$. If $\varphi \in P$, then $m m^{*}(\varphi)=\iint \varphi\left(g h^{-1}\right) d m(g) d \bar{m}(h) \leqq$ $\varphi(0)\left(\int_{G} d|m|(g)\right)^{2}=\|m\|^{2} e(\varphi)$, where $\|m\|$ denotes the total variation of $m$, and $e(\varphi)=\varphi(0)$. Since $\varphi$ is arbitrary in $P$, it follows that $m m^{*} \leqq\|m\|^{2} e$. Since $B$ is bounded is $E^{(0) \prime}$, there exists a constant $|B|$ such that $m \in B$ implies $\|m\| \leqq|B|$. Hence $m m^{*} \leqq|B|^{2} e$.

Suppose now that $B \subset E^{(k) \prime}$ where $k>0$. Then we know that there are a bounded subset $C$ of $E^{(0) \prime}$ and a finite subset $D$ of $U^{(k)}$, respectively, such that $t \in B$ implies $t=\Sigma d_{i} m_{i}$, with $m_{i} \in C, d_{i} \in D$. Hence $t t^{*}=\left(\Sigma d_{i} m_{i}\right)\left(\Sigma d_{i} m_{i}\right)^{*} \leqq 2^{n} \Sigma\left(d_{i} m_{i}\right)\left(d_{i} m_{i}\right)^{*}$. But we also know that $m_{i} m_{i}{ }^{*} \leqq\left\|m_{i}\right\|^{2} e$, and hence that $\left(d_{i} m_{i}\right)\left(d_{i} m_{i}\right)^{*}=d_{i} m_{i} m_{i}{ }^{*} d_{i}{ }^{*} \leqq$ $\|m\|^{2} d_{i} d_{i}^{*}$. It follows that $t t^{*} \leqq 2^{n} \Sigma\left\|m_{i}\right\|^{2} d_{i} d_{i}^{*}$. Since $C$ is bounded in $E^{(0)}$, there is a constant $|C|$ such that $m \in C$ implies $\|m\| \leqq|C|$, and since $D$ is finite, we have $t t^{*} \leqq N 2^{N}|C|^{2} \sum_{d e D} d d^{*}$, where $N$ is the number of elements in $D$. Hence if $t \in B$, then $t t^{*}$ is bounded above by an element in $U^{(2 k)} \cap P^{\prime}$ which is independent of $t$, as required.

The converse, which we shall not need here, can be obtained by observing that every subset $B$ with the properties of the theorem is necessarily bounded on bounded subsets of $P$, and hence on $E$.

It follows from this result that every positive linear functional on $E^{\prime}$ is bounded on the bounded subsets of $E^{\prime}$. Hence we have.

Corollary 2. Every positive linear functional defined on $E^{\prime}$ is necessarily continuous, i.e., is defined by an element of $E$.

The moment problem. We are now in a position to take up the moment problem. First we recall that if $\varphi$ is a $C^{\infty}$ function of positive type on $G$, then $\varphi$ defines a positive linear functional on $E^{\prime}$. In particular, $\varphi$ defines a linear functional on $U$ which is positive on $U \cap P^{\prime}$.

Suppose now that we have a linear functional on $U$. Under what conditions is it defined by a $C^{\infty}$ function of positive type on $G$ ? A necessary condition is that the functional be positive on $U \cap P^{\prime}$. 
Our solution to the moment problem shows that this necessary condition is also sufficient. The proof is patter ned after refernce [1].

THEOREM 3. Every linear functional on the algebra $U$ which is positive on $U \cap P^{\prime}$ admits an extension to a linear functional on the algebra $E^{\prime}$ which is positive on $P^{\prime}$, and hence is defined by a $C^{\infty}$ function of positive type on $G$.

Proof. Let $\theta$ be any linear functional on $U$ which is positive on $U \cap P^{\prime}$. Define the sublinear functional $p$ on $H^{\prime}$ according to the formula $p(t)=$ inf. $\left\{\theta(d): d \in U \cap H^{\prime}\right.$ and $\left.t \leqq d\right\}$. Then from Lemma 1 we see that $p(t)$ is defined for all $t$ in $H^{\prime}$, and $p$ is obviously sublinear. Since $\theta(d)=p(d)$ for all $d$ in $U \cap H^{\prime}$, we know from the Hahn-Banach theorem that $\theta$ admits an extension to a linear functional $\theta^{-}$, defined on all of $H^{\prime}$, such that $\theta^{-}(t) \leqq p(t)$. If $-t \in P^{\prime}$, then $p(t) \leqq 0$, so $\theta^{-}(t) \leqq 0$, and $\theta^{-}$is positive on $P^{\prime}$. By complex linearity $\theta^{-}$now admits a unique extension to all of $E^{\prime}$. According to Corollary 2, $\theta^{-}$is automatically continuous on $E^{\prime}$, and hence is defined by an element $\varphi$ of $P: \theta^{-}(t)=t(\varphi)$.

Unitary representations. To obtain a solution of the Hamburger moment problem in its original form when $G$ is in the real line, we have only to pass to the dual group via the (inverse) Fourier transform. In fact, we can carry out this procedure whenever $G$ is Abelian. In the general case, however, the dual group must be replaced with the dual space of all irreducible unitary representations of $G$.

Every continuous representation $\rho$ of $G$ as a group of unitary operators on a Hilbert space $H$ defines a continuous *-representation $\rho$ of $E^{\prime}$ as a *algebra of unbounded operators on a certain invariant dense subspace $D$ of $H$ [8]. If an element $t$ of $E^{\prime}$ is of positive type, then $(\rho(t) v, v) \geqq 0$ for all $v \in D$, so that $\rho(t)$ is a positive operator on $D$. Hence if $v$ is any vector in $D$, then the linear functional $\theta$ determined by $v$ according to the formula $\theta(t)=(\rho(t) v, v)$ is positive on $E^{\prime}$, and so is defined by a function $\varphi$ of positive type in $E$. Conversely, if $\varphi$ is such a function, then we know that there exists a unitary representation $\rho$ of $G$ on a Hilbert space $H$, and a vector $v$ in $H$ such that the positive functional $\theta$ defined by $\varphi$ on $E^{\prime}$ is determined by $v$ [7]. This leads us to the following restatement of Theorem 3:

THEOREM 4. Every linear functional on the algebra $U$ which is positive on all elements whose representatives are positive in each irreducible unitary representation of $G$ is determined by a vector in 
some unitary representation of $G$.

Not all *-representations of $U$ are determined by unitary representations of $G$ in the manner described above [8]. Those which are must evidently be completely reducible, and must represent elements of positive type by positive operators. Theorem 3 enables us to conclude that these necessary conditions are also sufficient:

THEOREM 5. Every completely reducible *-representation of $U$ in which elements of positive type are represented by positive operators is defined by a unitary represesentation of $G$ in the manner described above.

REMARKs. It is remarkable that the hypothesis of Theorem 3 apparently involves only the local structure of the group $G$, while the conclusion evidently involves the global structure. In fact, however, the global structure of $G$ is reflected in the order structure of the associated enveloping algebra $U$. Thus, distinct locally isomorphic Lie groups, having the same Lie algebra, will define different positive cones in the enveloping algebra. Hence the notion of positive type depends upon the group $G$ as well as upon the enveloping algebra $U$. This curious fact can be verified directly for the locally isomorphic Abelian groups $R$ and $T=R / Z$.

It would be desirable for applications to obtain an intrinsic characterization of the elements of positive type in the enveloping algebra. We know that every element of the form $t t^{*}$ lies in $U \cap P^{\prime}$. If $G=R$, elements of this form exhaust $U \cap P^{\prime}$. If $G=R^{2}$, however, convex combinations of elements of this form are not even dense in $U \cap P^{\prime}$ [5, p. 232], and no reasonable characterization of $U \cap P^{\prime}$ is known.

In general, the function of positive type obtained in Theorem 3 is not unique, even when $G=R$. In order to ensure uniqueness, we must require in addition that the linear functional on $U$ be not too badly unbounded. The following criterion, familiar in the classical problem, can be derived from the work of Nelson [8, section 9].

Let $d_{i} \cdots, d_{n}$ denote here a complete set of generators for the Lie algebra $g$, and put $\Delta=\Sigma d_{i}{ }^{2}$. Then $\Delta$ lies in $U \cap P^{\prime}$, and if $\theta$ is any positive linear functional on $U$ then the moments $\theta\left(\Delta_{n}\right)$ of $\Delta$ are all $\geqq 0$. If in addition these moments are all such that the series $\Sigma \lambda^{n} \theta\left(\Delta^{n}\right) / n$ ! converges for some real $\lambda$, then we say the functional $\theta$ is analytic on $U$. Every analytic positive functional on $U$ admits a unique extension to an analytic positive functional on $E^{\prime}$ which is defined by an analytic function of positive type in $E$. [8]. 


\section{REFERENCES}

1. F. F. Bonsall, Regular ideals of partially ordered vector spaces, Proc. London Math. Soc., (3) 6 (1956), 626-640.

2. G. deRham, and K. Kodaira, Harmonic Integrals, Mimeographed Lecture Notes, Institute for Advanced Study (1950).

3. J. Eells, Geometric Aspects of Currents and Distributions, Proc. Math. Acad. Sci. 41 (1955), 493-496.

4. L. Ehrenpreis, Some properties of distributions on Lie groups, Proc. J. Math., 6 (1956), 591-605.

5. I. M. Gelfand, and N. Y. Vilenkin, Generalized Functions, Vol. 4, Academic Press, New York, 1964.

6. H. Hamburger, Ueber eine Erweiterung des Stieltjes' schen Momenten Problems, Math. Ann., 81 (1920), 235-319.

7. L. Loomis, An Introduction to Abstract Harmonic Analysis, van Nostrand, New York, 1953.

8. E. Nelson, Analytic Vectors, Ann. Math., 70 (1959), 572-615.

9. L. Schwartz, Théorie des Distributions, Vols. I, II, Hermann, Paris, 1950-51.

10. J. A. Shohat, and J. D. Tamarkin, The Problem of Moments, Amer. Math. Soc., New York, 1943.

11. F. Treves, Topological Vector Spaces, Distributions and Kernels, Academic Press, New York, 1967.

Received April 14, 1970.

Dartmouth College 



\section{PACIFIC JOURNAL OF MATHEMATICS}

\section{EDITORS}

\author{
H. SAMELSON \\ Stanford University \\ Stanford, California 94305 \\ C. R. HовBY \\ University of Washington \\ Seattle, Washington 98105
}

J. DUGUndis

Department of Mathematics

University of Southern California

Los Angeles, California 90007

RICHARD ARENS

University of California

Los Angeles, California 90024

\section{ASSOCIATE EDITORS}
E. F. BeCKenbaCH
B. H. NeUmanN
F. WOLF
K. YoshidA

\section{SUPPORTING INSTITUTIONS}

\author{
UNIVERSITY OF BRITISH COLUMBIA \\ CALIFORNIA INSTITUTE OF TECHNOLOGY \\ UNIVERSITY OF CALIFORNIA \\ MONTANA STATE UNIVERSITY \\ UNIVERSITY OF NEVADA \\ NEW MEXICO STATE UNIVERSITY \\ OREGON STATE UNIVERSITY \\ UNIVERSITY OF OREGON \\ OSAKA UNIVERSITY \\ UNIVERSITY OF SOUTHERN CALIFORNIA
}

\author{
STANFORD UNIVERSITY \\ UNIVERSITY OF TOKYO \\ UNIVERSITY OF UTAH \\ WASHINGTON STATE UNIVERSITY \\ UNIVERSITY OF WASHINGTON

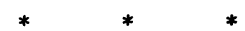 \\ AMERICAN MATHEMATICAL SOCIETY \\ CHEVRON RESEARCH CORPORATION \\ NAVAL WEAPONS CENTER
}

The Supporting Institutions listed above contribute to the cost of publication of this Journal, but they are not owners or publishers and have no responsibility for its content or policies.

Mathematical papers intended for publication in the Pacific Journal of Mathematics should be in typed form or offset-reproduced, (not dittoed), double spaced with large margins. Underline Greek letters in red, German in green, and script in blue. The first paragraph or two must be capable of being used separately as a synopsis of the entire paper. The editorial "we" must not be used in the synopsis, and items of the bibliography should not be cited there unless absolutely necessary, in which case they must be identified by author and Journal, rather than by item number. Manuscripts, in duplicate if possible, may be sent to any one of the four editors. Please classify according to the scheme of Math. Rev. Index to Vol. 39. All other communications to the editors should be addressed to the managing editor, Richard Arens, University of California, Los Angeles, California, 90024.

50 reprints are provided free for each article; additional copies may be obtained at cost in multiples of 50 .

The Pacific Journal of Mathematics is published monthly. Effective with Volume 16 the price per volume (3 numbers) is $\$ 8.00$; single issues, $\$ 3.00$. Special price for current issues to individual faculty members of supporting institutions and to individual members of the American Mathematical Society: $\$ 4.00$ per volume; single issues $\$ 1.50$. Back numbers are available.

Subscriptions, orders for back numbers, and changes of address should be sent to Pacific Journal of Mathematics, 103 Highland Boulevard, Berkeley, California, 94708.

PUBLISHED BY PACIFIC JOURNAL OF MATHEMATICS, A NON-PROFIT CORPORATION

Printed at Kokusai Bunken Insatsusha (International Academic Printing Co., Ltd.), 7-17, Fujimi 2-chome, Chiyoda-ku, Tokyo, Japan. 


\section{Pacific Journal of Mathematics}

Vol. 38, No. $2 \quad$ April, 1971

Richard Davis Anderson and Thomas Ashland Chapman, Extending

homeomorphisms to Hilbert cube manifolds .................. 281

Nguyen Huu Anh, Restriction of the principal series of $\operatorname{SL}(n, \mathbf{C})$ to some

reductive subgroups................................ 295

David W. Boyd, Indices for the Orlicz spaces . . . . . . . . . . . . 315

William Garfield Bridges, The polynomial of a non-regular digraph ...... 325

Billie Chandler Carlson, Robert K. Meany and Stuart Alan Nelson, Mixed

arithmetic and geometric means........................ 343

H. A. Çelik, Commutative associative rings and anti-flexible rings ...... 351

Hsin Chu, On the structure of almost periodic transformation groups ...... 359

David Allyn Drake, The translation groups of n-uniform translation

Hjelmslev planes ................................ 365

Michael Benton Freeman, The polynomial hull of a thin two-manifold . . . . 377

Anthony Alfred Gioia and Donald Goldsmith, Convolutions of arithmetic

functions over cohesive basic sequences .................... 391

Leslie C. Glaser, A proof of the most general polyhedral Schoenflies

conjecture possible ................................

Thomas Lee Hayden and Ted Joe Suffridge, Biholomorphic maps in Hilbert

space have a fixed point ................................ 419

Roger Alan Horn, Schlicht mappings and infinitely divisible kernels ...... 423

Norman Ray Howes, On completeness ...................... 431

Hideo Imai, Sario potentials on Riemannian spaces................ 441

A. A. Iskander, Subalgebra systems of powers of partial universal

algebras.

Barry E. Johnson, Norms of derivations of $\mathscr{L}(\mathrm{X})$.

David Clifford Kay and Eugene W. Womble, Axiomatic convexity theory and relationships between the Carathéodory, Helly, and Radon numbers

Constantine G. Lascarides, A study of certain sequence spaces of Maddox

and a generalization of a theorem of Iyer .............

C. N. Linden, On Blaschke products of restricted growth .

John S. Lowndes, Some triple integral equations ................. 515

Declan McCartan, Bicontinuous preordered topological spaces ......... 523

S. Moedomo and J. Jerry Uhl, Jr., Radon-Nikodým theorems for the Bochner and Pettis integrals ...

Calvin Cooper Moore and Joseph Albert Wolf, Totally real representations

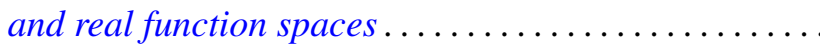

Reese Trego Prosser, A form of the moment problem for Lie groups. ... 